\title{
Role of High Frequency Oscillations of Somatosensory Evoked Potentials in Deciphering Pathophysiology of Migraine
}

\author{
Abhinandan K. Shankar ${ }^{1}$ Mahendra Javali1, Anish Mehta ${ }^{1} \quad$ R. Pradeep ${ }^{1} \quad$ Rohan Mahale $^{1}$ \\ Purushottam Acharya ${ }^{1}$ Rangasetty Srinivasa ${ }^{1}$
}

${ }^{1}$ Department of neurology, MS Ramaiah Medical College \& Hospital, Bengaluru, Karnataka, India

Address for correspondence Mahendra Javali, DM, Department of Neurology, M.S. Ramaiah Medical College \& Hospital, Bangalore, Karnataka 560054, India (e-mail: mahendrajv@gmail.com).

J Neurosci Rural Pract:2021;12:12-15

Abstract


Keywords
- thalamocortical drive
- habituation
- migraine
- evoked potentials
- latency
- amplitude

Background Habituation deficit is considered as a neurophysiological abnormality among migraineurs in the interictal period. For clear comprehension and clarity about the mechanism underlying habituation in migraine, a sophisticated method, i.e., high frequency oscillations (HFOs) evoked potentials, have been utilized. However, studies pertaining to this in the Indian context are rare.

Objective The aim of the study is to determine the utility of HFO of somatosensory evoked potential (SSEP) in deciphering the pathophysiology of migraine.

Materials and Methods Sixty subjects including 30 migraineurs in the interictal period and 30 healthy controls were considered for the study. Median nerve SSEP was recorded in patients and controls by standard protocols. HFO was extracted offline using the Digital zero-phase shift band-pass filtering at 450 and $750 \mathrm{~Hz}$. The early and late HFOs were determined with respect to the N20 peak and were compared between the groups. Results Of total 30 migraineurs, 18 had hemicranial headache and 12 had holocranial headache. N20 latency, P25 latency, N20 onset to peak amplitude, and N20 onset to P25 amplitude were comparable in migraineurs and controls. The intraburst frequency of early HFOs in migraineurs was significantly higher $(p=0.04)$, whereas the peak-to-peak amplitude was significantly lower $(p=0.001)$.

Conclusion Early HFOs on SSEP represent the thalamocortical excitatory drive in migraineurs. Overall, the study reports that reduced amplitude of early HFOs in the interictal period suggest reduced thalamocortical drive in migraineurs.

\section{Introduction}

Migraine is a complex neurological condition characterized by recurring episodes of headache with a prevalence of 8 to $15 \%$. Most often, migraine headache is unilateral and can be a cause of nausea and visual or sensory disturbances (aura). ${ }^{1}$ The migraineur's brain has high susceptibility to light stimuli and acoustic noise, which might be because of lack of habituation. ${ }^{2,3}$ The lack of habituation can be demonstrated on the scalp-recorded visual evoked potentials or somatosensory evoked potentials (SSEPs) after repeated stimuli. ${ }^{4}$ Although various hypotheses have been proposed, the exact etiopathogenesis of underlying migraine attacks still remains indistinct. ${ }^{5}$

Recent analysis has proposed migraine as a brain function disorder rather than vascular disease. ${ }^{5,6}$ Cortical hyperactivity induced by external stimuli is considered as the reason behind migraine headaches. ${ }^{7}$ Various neurophysiological methods are proposed to measure the in vivo electrocortical
DOI https://doi.org/ 10.1055/s-0040-1716793 ISSN 0976-3147.
(C) 2020. Association for Helping Neurosurgical Sick People.

This is an open access article published by Thieme under the terms of the Creative Commons Attribution-NonDerivative-NonCommercial-License, permitting copying and reproduction so long as the original work is given appropriate credit. Contents may not be used for commercial purposes, or adapted, remixed, transformed or built upon. (https://creativecommons.org/licenses/by-nc-nd/4.0/)

Thieme Medical and Scientific Publishers Pvt. Ltd. A-12, 2nd Floor, Sector 2, Noida-201301 UP, India 
responses to external sensory stimuli in migraine patients. It was found that altered thalamocortical connections with cortical dysexcitability may result in lack of habituation in migraineurs' brains in response to external stimuli. ${ }^{6}$ The abnormal information processing rises during pain-free days and reaches maximum before the attacks and vanishes in the ictal phase. ${ }^{8}$

For clear comprehension and clarity about the mechanism underlying the habituation in migraine, a sophisticated method, i.e., high frequency oscillations (HFOs) evoked potentials have been utilized. ${ }^{5}$ HFOs reflect the activity of an arousal system devoted to enhance the efficacy of the somatosensory input processing. Based on the theoretical origin, the scalp-recorded HFOs are of two types-early HFOs (former burst) generated in thalamocortical radiations and late HFOs (latter bursts) generated in cortical neurons at postsynaptic zone. ${ }^{9}$ Therefore, HFOs obtained using standard SSEP depict the thalamocortical drive in migraineurs brain. $^{5}$

Several studies have recently been performed to assess the utility of HFOs in interpreting the etiopathogenesis of migraine, and provide thalamocortical drive alterations as a neurophysiological sign of migraine. ${ }^{5,6,10,11}$ However, studies evaluating HFOs using SSEPs in migraine in the Indian context are quite rare. The study was thus intended to evaluate the usefulness of HFOs using median nerve SSEP in deciphering the pathophysiology of migraine.

\section{Materials and Methods}

This prospective, single-center, hospital-based case-control study (June 2016-November 2016) was conducted at the Department of Neurology. Institutional Scientific Committee and Ethics Review Board approval was obtained before the study initiation. Consecutive patients presenting with migraine during interictal period defined according to ICHDIII criteria, aged more than 18 years and treatment naïve were included. Thirty age- and sex-matched healthy participants without family history of migraine were included as controls. All pregnant women and subjects with secondary headaches were excluded. Informed consent was sought from both patients and controls before the study initiation.

Demographic characteristics, frequency of headache, topography of headache, duration of each attack, migraine with or without aura, precipitating factors, family history, and comorbidities of the patients were recorded.

Subjects were made to relax on a comfortable chair in an illumined room with eyes open and concentrate on the wrist movement. Later, active electrodes were positioned on the contralateral parietal area ( $\mathrm{C}^{\prime}, 2-\mathrm{cm}$ posterior to $\mathrm{C} 3$ in the international 10-20 systems) and on the fifth cervical spinous process (Cv5) and ground electrode on the right arm. The electrical stimulation was then delivered to the right median nerve in the wrist for 0.2 milliseconds using a square wave pulse constantly with stimulus intensity twice the motor threshold to record SSEPs. The recording was done at least 3 days before or after their last or their next migraine attack.
Thereafter, digital zero-phase shift band-pass filtering (Barlett-Hanning window, 51 filter coefficients) was applied off-line between 450 and $750 \mathrm{~Hz}$ to extract HFOs superimposed on the N20 left parietal component of the SSEP. From the wide-band unfiltered SSEPs $(0-2,500 \mathrm{~Hz})$, various components respective to their latencies (N13, N20, P25, and N33) were identified. Peak-to-peak (pk-pk) amplitudes of the $\mathrm{N} 13$ cervical component under the $\mathrm{Cv} 5$ active derivation and of N20 onset-N20 peak, N20peak-P25, P25-N33 under the active C3' left-sided scalp electrode was recorded. The HFOs bursts were identified with regard to the N20 onset on the wide-band pass SSEP trace. The initiation of burst was measured from the first wavelet having amplitude 50\% greater than the electrical noise. Termination of the burst was measured as the last wavelet having amplitude 50\% greater than the electrical noise. The HFOs burst appeared as a train of approximately 5 to 8 wavelets, was classified into two segments based on N20 peak as early and late HFOs. The early and later HFOs bursts appeared in the ascending and descending slope of N20 component. The frequency of early HFOs was more than the late HFOs, whereas frequency and amplitude between two bursts were declined ( $\boldsymbol{- \text { Fig. }}$ 1). If the difference between two HFOs was not possible, then the burst occurring earlier and later the N20 peak was considered to categorize early and late HFOs. The latency of the maximum negative peak, intraburst frequency, number of negative peaks, and the maximum pk-pk amplitude on the two HFO bursts were separately measured. A sample tracing of HFO recording of our study is as shown in - Fig. 2 .

\section{Statistical Analysis}

SPSS 17 (IBM. Inc., Texas, United States) software was used to analyze the data. Categorical data were expressed either in percentages or frequency while continuous data in mean \pm SD. Wilcoxon rank sum test was applied on HFOs parameters to study the difference and similarity in normal and migraine. A $p$-value $(\leq 0.05)$ was regarded as significant.

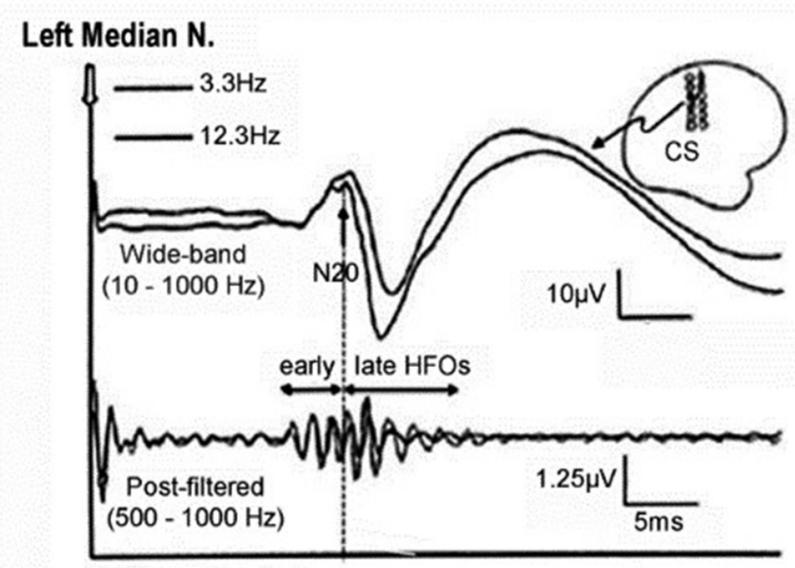

Fig. 1 An illustration of wide-band SSEP and post-filtered high frequency oscillations of left median nerve. SSEP, somatosensory evoked potential. 


\section{Results}

SSEP recordings were taken from all patients and controls. The mean age of the migraineurs was $32.2 \pm 5.6$ years and of controls was $29.4 \pm 4.8$ years $(p=0.42)$. Out of 30 migraineurs, 12 were men and 18 were women. Out of 30 controls, 16 were men and 14 were women. The mean duration of having history of headache was $3.2 \pm 1.4$ years. Of total 30 migraineurs, 18 had hemicranial headache and 12 had holocranial headache. Four patients had visual aura prior to headache episode. A family history of migraine was noted in ten patients.

N20 latency, P25 latency, N20 onset to peak amplitude, and N20 onset to P25 amplitude were comparable in migraineurs and controls ( - Table $\mathbf{1}$ ).

The intraburst frequency of early HFOs in migraineurs was significantly higher compared with controls $(p=0.04)$.
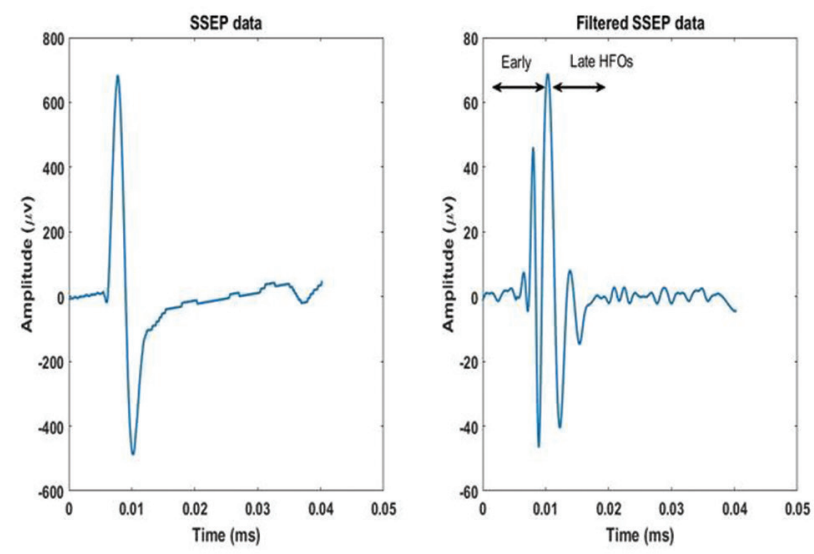

Fig. 2 An illustrative sample of SSEP data and filtered SSEP data of a patient with migraine. SSEP, somatosensory evoked potential.

Table 1 Latencies and amplitudes of wide-bandpass SSEPS

\begin{tabular}{|l|l|l|l|}
\hline Evoked response & Controls & Migraine & $p$-Value \\
\hline N20 & $22.17 \pm 5.11$ & $21.75 \pm 1.75$ & 0.98 \\
\hline P25 & $28.70 \pm 4.28$ & $26.87 \pm 3.08$ & 0.85 \\
\hline N20onset-N20peak & $1.17 \pm 0.46$ & $1.42 \pm 0.23$ & 0.10 \\
\hline N20peak-P25 & $2.39 \pm 0.54$ & $2.45 \pm 0.44$ & 0.86 \\
\hline
\end{tabular}

Abbreviations: $N$, negative; $P$, positive; SSEP, somatosensory evoked potential.

Note: Data expressed as mean \pm SD.
The maximum pk-pk amplitude of the first burst of HFOs was significantly smaller in migraineurs than in controls $(p=0.001)$. The latency of the maximum negative peak, intraburst frequency, number of negative peaks, and maximum pk-pk amplitude for late HFO bursts were similar between the two groups as shown in -Table 2 .

\section{Discussion}

HFO is a relatively novel method that explores both the excitatory and inhibitory mechanisms simultaneously of the central nervous system. It has been broadly used to identify several neurological disorders such as Parkinson's disease, multiple system atrophy, myoclonus epilepsy, etc. ${ }^{11}$ Hence, in our study, we utilized HFOs of SSEP in interpreting the pathophysiology of migraine disorder.

Literature has reported that a strong relationship exists between clinical and neurophysiological profiles in migraine. ${ }^{6}$ Various studies reported that the amplitude of early HFOs significantly reduces in migraineurs, suggesting a reduced thalamocortical excitatory drive, resulting in the habituation deficit in migraine. ${ }^{5-7}$ In this study as well, amplitude of early HFOs reduced in migraineurs in the interictal period, reflecting reduced thalamocortical drive.

Most migraineurs in this study were aged 32 years, without any treatment history, indicating that the patients were in quite the early stages of migraine. In this study, an insignificant difference in broad-band conventional SSEPs between migraineurs and controls suggests normal relay of somatosensory signal along the posterior columns up to contralateral somatosensory cortex. Other studies too have reported that broad-band SSEP was similar in both migraineurs and controls.,12

Research has reported that reduced amplitude of early HFOs in migraineurs between attacks advocates lack of habituation. ${ }^{11}$ The habituation deficit in migraineurs may be due to diminished activity of inhibitory interneurons in the neocortex and/or a decreased activity of thalamocortical projections. The reduced early HFOs in migraineurs suggest that migraine headaches might occur due to reduced thalamocortical excitatory cholinergic afferent functions. It was also demonstrated that the lack of difference in late HFOs between migraineurs and controls suggests normal functioning of cortical inhibitory interneurons and pyramidal chattering cells in migraineurs. ${ }^{12,13}$

Table 2 Electrophysiological parameters of early and late HFOs

\begin{tabular}{|l|l|l|l|l|l|l|}
\hline \multirow{2}{*}{} & \multicolumn{2}{|c|}{ First phase (early HFO) } & \multirow{2}{*}{$p$-Value } & \multicolumn{2}{c|}{ Second phase (late HFO) } & $p$-Value \\
\cline { 2 - 6 } & Control & Migraine & & Control & Migraine \\
\hline $\begin{array}{l}\text { Latency of maximum negative } \\
\text { peak }(\mathrm{ms})\end{array}$ & $16.23 \pm 7.84$ & $15.0 \pm 3.66$ & 0.85 & $46.94 \pm 10.21$ & $50.68 \pm 3.28$ & 0.73 \\
\hline Intraburst frequency & $533.64 \pm 135.21$ & $614 \pm 88.87$ & $0.04^{\mathrm{a}}$ & $334.29 \pm 85.29$ & $279.18 \pm 15.25$ & 0.06 \\
\hline Number of negative peaks & $2.0 \pm 0.3$ & $1.95 \pm 0.44$ & 0.07 & $2.17 \pm 0.52$ & $2 \pm 0.73$ & 0.26 \\
\hline $\begin{array}{l}\text { Maximum peak-to-peak } \\
\text { amplitude }(\mu \mathrm{V})\end{array}$ & $0.068 \pm 0.015$ & $0.042 \pm 0.0351$ & $0.001^{\mathrm{a}}$ & $0.039 \pm 0.025$ & $0.038 \pm 0.0140$ & 0.71 \\
\hline
\end{tabular}

Abbreviation: HFO, high frequency oscillations.

${ }^{a} p$-value $<0.05$ is significant.

Note: Data expressed as mean \pm SD. 
In this study, abnormalities in the early HFOs were found as mitigated amplitude with increased intraburst frequency as compared with controls, while no abnormalities were noted in late HFOs. A similar study by Sakuma et $\mathrm{al}^{14}$ reported comparable amplitudes and peak latencies for N9, N13, N20, and P25 components in both migraineurs and controls. However, root-mean-square amplitudes of HFOs in the migraineurs were diminished significantly as compared with controls. ${ }^{14}$ A study by Coppola et al ${ }^{12}$ reported lower maximum pk-pk amplitude and area under rectified curve in early HFO burst in migraineurs compared with healthy volunteers, whereas no such differences were reported in late HFO burst. It was also postulated that thalamocortical activation (early HFOs) reduces during the interictal period and normalizes during the migraine attack, whereas late HFOs remain the same during or between the attacks. ${ }^{12}$ A study by Restuccia et al ${ }^{15}$ demonstrated that the amplitude of early presynaptic HFOs correlates significantly with the clinical evolution, while postsynaptic HFOs did not correlate with clinical fluctuations. It was also reported that spontaneous worsening reduces presynaptic HFOs while spontaneous improvement enhances presynaptic HFOs. ${ }^{15}$ Coppola et $a{ }^{6}$ reported that pk-pk amplitudes of late HFOs significantly increase in chronic migraine and migraine during the attack compared with healthy volunteers and migraineurs in between the attacks. ${ }^{6}$

Furthermore, insignificant negative peaks were lesser in early HFOs in migraineurs than controls, indicating weakened early HFOs in between the attacks. A similar study by Prakash $^{5}$ also stated that decreased activity of thalamocortical excitatory cholinergic afferents results in subcortical hypoexcitability in migraineurs in between attacks.

The limitation of the study was the small size of the sample. We did not perform similar recordings in patients with other primary headaches like tension-type headache which would verify the specificity of our verdicts in migraine. We also did not perform recordings in patients who were on prophylactic antimigraine drugs to demonstrate the reversal of abnormalities as compared with the drug naïve patients.

\section{Conclusion}

Deficit in the habituation is the primary abnormality in migraine, responsible for hyper-responsivity of cerebral cortex. Habituation is due to the normal thalamocortical excitatory drive. Early HFOs on SSEP represent the thalamocortical excitatory drive in migraineurs. Overall, the study reports that reduced amplitude of early HFOs in the interictal period suggests reduced thalamocortical drive in migraineurs.

\section{Authors' Contributions}

A. K. S. conceptualized, designed, provided definition of intellectual content, performed literature search, analyzed data and statistics, assisted in manuscript preparation, and is a guarantor. M. J. conceptualized, designed, provided definition of intellectual content, assisted in manuscript preparation, manuscript editing, manuscript review, and is a guarantor. A. M. designed, performed literature search, reviewed manuscript, and is a guarantor. P. R., R. M., P. A., and R. S reviewed manuscript and are guarantors.

\section{Funding}

None.

\section{Conflict of Interest}

None declared.

\section{Acknowledgments}

The authors thank Mr. Raghu, PhD Scholar, for providing support during our research work.

\section{References}

1 Martelletti P, Birbeck GL, Katsarava Z, Jensen RH, Stovner LJ, Steiner TJ. The Global Burden of Disease survey 2010, lifting the burden and thinking outside-the-box on headache disorders. J Headache Pain 2013;14(1):13

2 Noseda R, Burstein R. Migraine pathophysiology: anatomy of the trigeminovascular pathway and associated neurological symptoms, cortical spreading depression, sensitization, and modulation of pain. Pain 2013;154(suppl 1) :S44-S53

3 Bohotin V, Fumal A, Vandenheede M, et al. Effects of repetitive transcranial magnetic stimulation on visual evoked potentials in migraine. Brain 2002;125(Pt 4) :912-922

4 Ambrosini A, Schoenen J. Electrophysiological response patterns of primary sensory cortices in migraine. J Headache Pain 2006;7(6):377-388

5 Prakash R. Somatosensory evoked high frequency oscillations in a homogeneous population of drug naive migraineurs. Int J Physiol 2020;8(1):62-67

6 Coppola G, Iacovelli E, Bracaglia M, Serrao M, Di Lorenzo C, Pierelli F. Electrophysiological correlates of episodic migraine chronification: evidence for thalamic involvement. J Headache Pain 2013;14(1):76

7 Ren J, Xiang J, Chen Y, Li F, Wu T, Shi J. Abnormal functional connectivity under somatosensory stimulation in migraine: a multi-frequency magnetoencephalography study. J Headache Pain 2019;20(1):3

8 Coppola G, Currà A, Di Lorenzo C, et al. Abnormal cortical responses to somatosensory stimulation in medication-overuse headache. BMC Neurol 2010;10(1):126

9 Kalita J, Bhoi SK, Misra UK. Effect of high rate rTMS on somatosensory evoked potential in migraine. Cephalalgia 2017;37(13):1222-1230

10 Coppola G, Pierelli F, Schoenen J. Habituation and migraine. Neurobiol Learn Mem 2009;92(2):249-259

11 Lai K-L, Liao K-K, Fuh J-L, Wang S-J. Subcortical hyperexcitability in migraineurs: a high-frequency oscillation study. Can J Neurol Sci 2011;38(2):309-316

12 Coppola G, Vandenheede M, Di Clemente L, et al. Somatosensory evoked high-frequency oscillations reflecting thalamo-cortical activity are decreased in migraine patients between attacks. Brain 2005;128(Pt 1):98-103

13 Restuccia D, Della Marca G, Valeriani M, et al. Influence of cholinergic circuitries in generation of high-frequency somatosensory evoked potentials. Clin Neurophysiol 2003; 114(8):1538-1548

14 Sakuma K, Takeshima T, Ishizaki K, Nakashima K. Somatosensory evoked high-frequency oscillations in migraine patients. Clin Neurophysiol 2004;115(8):1857-1862

15 Restuccia D, Vollono C, Del Piero I, Martucci L, Zanini S. Somatosensory high frequency oscillations reflect clinical fluctuations in migraine. Clin Neurophysiol 2012; 123(10):2050-2056 